\title{
Numerical ISS controller design via a dynamic game approach
}

\author{
Lars Grüne and Manuela Sigurani
}

\begin{abstract}
We present a numerical design method for an input-to-state practically stabilizing (ISpS) feedback controller for perturbed discrete time nonlinear control systems. By appropriately scaling the system, the problem of ISpS controller design can be converted into a uniform practical stabilization problem under perturbations which can be solved by a numerical dynamic game approach. We present and analyze this approach with a particular focus on a quantitative analysis of the resulting gain and the size of the exceptional region for practical stability.
\end{abstract}

\section{INTRODUCTION}

Since its introduction by Sontag in [22], the concept of input-to-state stability has become one of the most influential concepts in nonlinear stability under perturbations. Besides yielding a theoretically sound concept for the quantitative and qualitative analysis of stability of nonlinear systems under perturbations, one of its particular features is the possibility to analyze the stability of interconnected systems by means of analyzing low dimensional subsystems via ISS small gain arguments, see, e.g., [16], [2], [3]. The latter particularly allows for a rigorous approach to decentralized controller design by designing input-to-state stabilizing controllers. Hence, a systematic way to design ISS controllers can in particular serve as a building block for decentralized stabilizing feedback design for large networks of nonlinear systems.

Similar to stabilizing controllers for nonlinear systems, ISS controllers can in principle be derived from corresponding ISS Lyapunov functions via a universal or Sontag type formula [20]. However, applying this formula requires the analytic knowledge of an ISS Lyapunov function which may not always be available. An alternative are dynamic programming type design methods relying on optimal control formulations whichrequire much less a priory analytical knowledge. The main drawback of this approach is the curse of dimensionality which means that it is only computationally feasible for systems of moderate space dimension. However, in a small gain based decentralized setting in which the controller design is to be carried out for a set of low dimensional subsystems the approach may provide an attractive and feasible alternative, which is why we investigate it in this paper.

A dynamic programming based ISS controller design was

This work was supported by the DFG Priority Program 1305 and by the European Union under the 7th Framework Programme FP7-PEOPLE2010-ITN, Grant agreement nr. 264735-SADCO

L. Grüne and M. Sigurani are with the Mathematical Institute, University of Bayreuth, 95440 Bayreuth, Germany lars.gruene, manuela.sigurani@uni-bayreuth. de proposed in $[15]^{1}$. However, the approach in this paper has the drawback that by converting the problem into an auxiliary $\ell^{\infty}$ control problem the state variable needs to be augmented by 2 additional scalar states which considerably increases the computational complexity of the controller design. In order to avoid this problem, in this paper we propose an approach which consists of converting the ISS controller design problem into a uniform stabilization problem for a perturbed system which can be accomplished without increasing the dimension. The equivalence between ISS and robust stability was already exploited in a theoretical context in [17] (for a continuous time version of this result see [23]) and thus our approach can be seen as a constructive numerical interpretation of the results in [17]. In order to solve the auxiliary stabilization problem under perturbations we use the game theoretic algorithmic approach from [8] which in turn relies on [9], [19].

Like in most nonlinear numerical approaches relying on Lyapunov functions, cf. e.g., [4], [14], [5], a neighborhood of the equilibrium (in this paper always chosen as the origin) needs to be treated in a different way. In our setting this means that the resulting nonlinear controller will in general only yield input-to-state practical stability (ISpS), i.e., the closed loop system will have the ISS property only outside a neighborhood of the origin. For this reason, a substantial part of the analysis in this paper is devoted to keeping track of the size of this exceptional neighborhood in order to control the errors introduced by the numerical solution of the dynamic game problem. This allows us to identify conditions under which this neighborhood is small. Hence, a linearization based design could be used if desired in order to define an ISS controller also near the origin. Moreover, we note that both the Lyapunov function as well as the resulting optimal feedback law are piecewise constant and thus discontinuous in our approach, which is why we provide an analysis entirely avoiding continuity assumptions.

\section{Setting}

Our goal is to construct an input-to-state practically stabilizing (ISpS) controller for the controlled and perturbed discrete-time system

$$
x_{k+1}=f\left(x_{k}, u_{k}, w_{k}\right), \quad k=0,1, \ldots
$$

For simplicity of exposition, in this paper we consider a compact state space $X \subset \mathbb{R}^{d}$. Extensions to hybrid systems with state spaces containing discrete state components and

\footnotetext{
${ }^{1}$ This reference treats various different robust controller design objectives, among them ISS
} 
more general set stabilization problems can be addressed similarly to [7]. The discrete time model under consideration can, of course, be the discrete time representation of a sampled continuous time model.

The values $u_{k}$ and $w_{k}$ denote the control and perturbation acting on the system which are taken from sets $U \subset \mathbb{R}^{m}$ an $W \subset \mathbb{R}^{q}$, respectively, which again for simplicity of exposition are supposed to be compact. Infinite sequences of control and perturbation values are denoted by $\mathbf{u}=\left(u_{0}, u_{1}, \ldots\right)$ and $\mathbf{w}=\left(w_{0}, w_{1}, \ldots\right)$ and the corresponding spaces of such sequences with values $u_{k} \in U$ and $w_{k} \in W$ are denoted by $\mathscr{U}$ and $\mathscr{W}$, respectively.

The control objective of designing an ISpS controller means that we intend to find a static state feedback controller $u_{k}=u_{\mathscr{P}}\left(x_{k}\right)$ such that the closed loop system

$$
x_{k+1}=f\left(x_{k}, u_{\mathscr{P}}\left(x_{k}\right), w_{k}\right)=: h\left(x_{k}, w_{k}\right), \quad k=0,1, \ldots
$$

is input-to-state practically stable in the following sense.

Definition 1: System (2) is called input-to-state practically stable (ISpS) with respect to $\delta, \Delta_{w} \in \mathbb{R}_{\geq 0}$ on a set $Y \subset X$ if there exist $\beta \in \mathscr{K} \mathscr{L}$ and $\gamma \in \mathscr{K}$, such that the solutions of the system satisfy

$$
\left\|x_{k}\right\| \leq \max \left\{\beta\left(\left\|x_{0}\right\|, k\right), \gamma\left(\|\mathbf{w}\|_{\infty}\right), \delta\right\},
$$

for all $x_{0} \in Y$, all $\mathbf{w} \in \mathscr{W}$ with $\|\mathbf{w}\|_{\infty} \leq \Delta_{w}$ and all $k \in \mathbb{N}_{0}$.

The approach we present in this paper relies on the conversion of the ISpS controller design method into a uniformly practically stabilizing controller design method. To this end, in the next section we first sketch the dynamic game based uniformly practically stabilizing controller design method from [8]. Afterwards, we explain how to use this approach for the ISpS controller design problem at hand.

\section{GAME THEORETIC STABILIZING CONTROLLER DESIGN FOR PERTURBED SYSTEMS}

In this section we consider the perturbed control system

$$
x_{k+1}=\tilde{f}\left(x_{k}, u_{k}, d_{k}\right), \quad k=0,1, \ldots
$$

Except for changing the notation, this model has the same structure as (1). Moreover, $x_{k}, u_{k}$ and the respective sets and spaces remain unchanged. The perturbation values $d_{k}$ are now taken from a set $D \subset \mathbb{R}^{q}$, the corresponding sequences are denoted as $\mathbf{d}=\left(d_{0}, d_{1}, \ldots\right)$ and the space of such sequences with $d_{k} \in D$ is denoted by $\mathscr{D}$.

The control objective for System (4) is to design a practically uniformly stabilizing state feedback controller, i.e., a controller $u_{k}=u_{\mathscr{P}}\left(x_{k}\right)$ such that the closed loop system

$$
x_{k+1}=\tilde{f}\left(x_{k}, u_{\mathscr{P}}\left(x_{k}\right), d_{k}\right)=: \tilde{h}\left(x_{k}, d_{k}\right), \quad k=0,1, \ldots
$$

satisfies the following definition.

Definition 2: System (5) is called uniformly (w.r.t. $\mathbf{d} \in \mathscr{D}$ ) practically (w.r.t. $\delta$ ) asymptotically stable on a set $Y \subset X$ if there exist $\beta \in \mathscr{K} \mathscr{L}$ such that the solutions of the system satisfy

$$
\left\|x_{k}\right\| \leq \max \left\{\beta\left(\left\|x_{0}\right\|, k\right), \delta\right\},
$$

for all $x_{0} \in Y$, all $\mathbf{d} \in \mathscr{D}$ and all $k \in \mathbb{N}_{0}$.
In order to design a controller we employ the dynamic game approach from [8] which in turn relies on ideas from [9], [19]. For a given initial state $x \in X$, a given control sequence $\mathbf{u}=\left(u_{k}\right)_{k \in \mathbb{N}} \in \mathscr{U}$ and a given perturbation sequence $\mathbf{d}=\left(d_{k}\right)_{k \in \mathbb{N}} \in \mathscr{D}$, we denote the solution trajectory of (4) by $\left(x_{k}(x, \mathbf{u}, \mathbf{d})\right)_{k \in \mathbb{N}}$.

Specifying a target set $T$ (typically a small neighborhood of the origin) and a stage cost $g(x, u)$, we define the accumulated cost as

$$
J(x, \mathbf{u}, \mathbf{w})=\sum_{k=0}^{k(T, x, \mathbf{u}, \mathbf{d})} g\left(x_{k}(x, \mathbf{u}, \mathbf{d}), u_{k}\right),
$$

with $k(T, x, \mathbf{u}, \mathbf{d}):=\inf \left\{k \geq 0 \mid x_{k}(x, \mathbf{u}, \mathbf{d}) \in T\right\}$. Here we assume that the stage cost satisfies the following assumption.

Assumption 3: The stage cost $g$ penalizes the distance to 0, i.e., there exists $\underline{\alpha} \in \mathscr{K}_{\infty}$ such that

$$
g(x, u) \geq \underline{\alpha}(\|x\|)
$$

holds for all $x \in X, u \in U$.

Using the accumulated cost we now define a dynamic game by means of its upper value function

$$
V(x)=\sup _{\beta \in \mathscr{B}} \inf _{\mathbf{u} \in \mathscr{U}} J(x, \mathbf{u}, \beta(\mathbf{u})), \quad x \in X,
$$

Here, $\mathscr{B}$ denotes the set of all nonanticipating strategies $\beta$ : $\mathscr{U} \rightarrow \mathscr{D}$, i.e. all strategies $\beta$ satisfying $u_{k}=u_{k}^{\prime} \quad \forall k \leq K \quad \Rightarrow$ $\beta(\mathbf{u})_{k}=\beta\left(\mathbf{u}^{\prime}\right)_{k} \quad \forall k \leq K$ for any two control sequences $\mathbf{u}=$ $\left(u_{k}\right)_{k}, \mathbf{u}^{\prime}=\left(u_{k}^{\prime}\right)_{k} \in \mathscr{U}$. By standard dynamic programming arguments [1] one sees that this function fulfills and is uniquely determined by the optimality principle

$$
V(x)=\inf _{u \in U} \sup _{d \in D}\{g(x, u)+V(\tilde{f}(x, u, d))\}
$$

for $x \notin T$ together with the boundary condition $\left.V\right|_{T} \equiv 0$. This equation can also be written as

$$
V(x)=\inf _{u \in U}\left\{g(x, u)+\sup _{x^{\prime} \in \tilde{f}(x, u, D)} V\left(x^{\prime}\right)\right\} .
$$

Note that in (10) the parameterization of $\tilde{f}$ by $d$ is not needed any more since it is sufficient to know the set valued image $\tilde{f}(x, u, D)$.

This fact is exploited in the discretization proposed in [8]. In this discretization, the set $X$ is decomposed into a finite partition $\mathscr{P}$ of boxes or cells $P$ with pairwise disjoint interior and $\bigcup_{P \in \mathscr{P}} P=X$. Then, a graph theoretic representation of the dynamics on $\mathscr{P}$ is constructed. Since the model includes both control and perturbation, the resulting graph theoretic approximation takes the form of a hypergraph. Solving a generalized min-max shortest path problem on this hypergraph then yields an approximation $V_{\mathscr{P}}$ of $V$ which is constant on each element $P$ of the partition $\mathscr{P}$.

The central trick introduced in [8] in terms of stabilization is to interpret the discretization error introduced by the partition $\mathscr{P}$ as a perturbation and explicitly include it in the computation. This way, one can prove that the approximation $V_{\mathscr{P}}$ is not an arbitrary approximation to $V$ but that $V_{\mathscr{P}}$ is in fact a practical control Lyapunov function. While in [8] 
the discretization error is the only perturbation acting on the system, here we extend the setting by considering both the original perturbation $d$ and the discretization error as perturbations. The necessary changes in the statements and proofs of the results in [8] are straightforward and are hence omitted due to space constraints. The resulting theorem then reads as follows.

Theorem 4: ([8, Theorem 1]) Let $V$ denote the optimal value function of the dynamic game (8) and let $V_{\mathscr{P}}$ denote the approximate optimal value function constructed according to the algorithm presented in [8] on a given partition $\mathscr{P}$ with target set $T \subset \mathscr{P}$ and $0 \in T$. Then,

$$
V(x)-\max _{y \in T} V(y) \leq V_{\mathscr{P}}(x),
$$

i.e. $V_{\mathscr{P}}$ is an upper bound for $V-\left.\max V\right|_{T}$. Furthermore, $V_{\mathscr{P}}$ satisfies

$$
V_{\mathscr{P}}(x) \geq \min _{u \in U}\left\{g(x, u)+\sup _{x^{\prime} \in \tilde{f}(x, u, D)} V_{\mathscr{P}}\left(x^{\prime}\right)\right\}
$$

for all $x \in X \backslash T$.

Note that $V_{\mathscr{P}}$ may assume the value $+\infty$ on some parts of $X$, in which case inequality (12) does not yield valuable information. We define the stabilizable set w.r.t. $V_{\mathscr{P}}$ by

$$
S_{\mathscr{P}}:=\left\{x \in X \mid V_{\mathscr{P}}(x)<\infty\right\} .
$$

Theorem 4 now motivates the definition of the controller

$$
u_{\mathscr{P}}(x):=\underset{u \in U}{\operatorname{argmin}}\left\{g(x, u)+\sup _{x^{\prime} \in \tilde{f}(x, u, D)} V_{\mathscr{P}}\left(x^{\prime}\right)\right\}
$$

for $x \in S_{\mathscr{P}} \backslash T$. For $x \in T$ we may either use Formula (13) or we may define $u_{\mathscr{P}}$ in a different way, e.g., constantly equal to 0 or by using linearization at the origin, as, e.g., proposed in [6] in an event based context. For $x \in X \backslash S_{\mathscr{P}}$, our approach does not allow for a meaningful definition of $u_{\mathscr{P}}$.

The following theorem from [8] summarizes the properties of $u_{\mathscr{P}}$.

Theorem 5: ([8, Theorem 2]) Consider system (4), a target set $T \subset \mathscr{P}$ with $0 \in T$ and the approximate optimal value function $V_{\mathscr{P}}$ constructed according to the algorithm presented in [8] on a given partition $\mathscr{P}$. For $x_{0} \in S_{\mathscr{P}}$ denote the trajectory of the closed loop system (5) with feedback $u=u_{\mathscr{P}}$ from (13) by $x_{k}$.

Then there exists $k^{*} \in \mathbb{N}$ such that

$$
\begin{gathered}
V_{\mathscr{P}}\left(x_{k}\right) \geq g\left(x_{k}, u_{\mathscr{P}}\left(x_{k}\right)\right)+V_{\mathscr{P}}\left(x_{k+1}\right), k \in\left\{0, \ldots, k^{*}-1\right\}, \\
x_{k^{*}} \in T \quad \text { and } \quad x_{k} \in N, k>k^{*}
\end{gathered}
$$

where $\quad N:=\left\{x \in X: V_{\mathscr{P}}(x) \leq v\right\}$ and $\quad v:=$ $\max _{x \in T, d \in D} V_{\mathscr{P}}\left(f\left(x, u_{\mathscr{P}}(x), d\right)\right)$, i.e., the closed loop trajectory enters the target set $T$ at time $k^{*}$ and stays in the sublevel set $N$, afterwards.

Remark 6: Note that it is in general not possible to use the target set $T=\{0\}$ unless we are willing to assume that the system can be controlled to the origin on finitely many steps (and even then using $T=\{0\}$ is likely to cause numerical problems). Similar problems in small neighborhoods of the equilibrium occur in many other numerical approaches for computing Lyapunov functions for nonlinear systems, even for non-controlled systems, see [4], [14], [5]. This means that on a small neighborhood around the origin $V_{\mathscr{P}}$ is not a Lyapunov function and consequently the exceptional set $N$ cannot be avoided. The size of this set then determines the size of the parameter $\delta$ on the practical stability definition.

In control problems, the usual way to work around this problem is to use linearization techniques in order to solve the feedback stabilization problem locally near 0 . For this purpose it is of utmost importance to keep the size of the exceptional set $N$ and thus of $\delta$ small. Consequently, one of the central tasks in the following section will be to carefully estimate this value in the ISpS context.

\section{ISPS CONTROLLER DESIGN}

In order to apply the algorithm from the previous section to practical ISS controller design we make use of one of the central results in [17], which states that System (2) is ISS if and only if it is robustly stable, i.e., if there exists $e: \mathbb{R}^{n} \times \mathbb{R}^{q} \rightarrow \mathbb{R}^{q}$ and $\eta \in \mathscr{K}_{\infty}$ such that System (5) with

$$
\tilde{f}(x, u, d)=f(x, u, e(x, d)) \quad \text { and } \quad D=\bar{B}_{1}(0)
$$

is uniformly asymptotically stable, where $e$ is such that for each $w \in W$ with $\|w\| \leq \eta(\|x\|)$ there exists $d \in D$ with $e(x, d)=w$. For instance, $e$ could be defined as $e(x, d):=$ $\eta(\|x\|) d$ which is also the choice in [17]. The proof of the equivalence between ISS and robust stability relies on Lyapunov function arguments.

In order to carry over the proof to our setting of practical stability, in this section we first provide a characterization of ISpS by means of an appropriate Lyapunov function $V$. In contrast to the analogous non-practical statements in [17], here we give a direct proof which allows to determine the resulting gains and the size of the practical stability region. Afterwards we then show that $V=V_{\mathscr{P}}$ is an ISpS Lyapunov function in our sense for the non-scaled system.

A particular difficulty in this derivation is the fact that in general neither $V_{\mathscr{P}}$ nor $u_{\mathscr{P}}$ are continuous. Hence, we will need to avoid all arguments in the proof which require continuity of $V_{\mathscr{P}}$ or $u_{\mathscr{P}}$. We begin by introducing a continuity condition on $f$ and a boundedness condition on $h$.

Assumption 7: The map $f: X \times U \times W \rightarrow \mathbb{R}^{n}$ in (1) is uniformly continuous in $w$ in the following sense: there exist $\gamma_{w} \in \mathscr{K}_{\infty}$, such that for all $x \in X, u \in U$ and $w \in W$

$$
\|f(x, u, w)-f(x, u, 0)\| \leq \gamma_{w}(\|w\|) .
$$

Since we assume $X$ and $U$ to be compact it is not very restrictive to assume that $\gamma_{w}$ is independent of $x$ and $u$. If needed, the inequality could be suitably generalized.

Assumption 8: The map $h: X \times W \rightarrow \mathbb{R}^{n}$ in (2) is bounded for $w=0$ on $T$ in the following sense: there exists $\gamma_{x} \in \mathscr{K}_{\infty}$ such that for all sufficiently small sets $T \subset X$ with $0 \in T$ and each $x \in T$ we have

$$
\|h(x, 0)\| \leq \gamma_{x}(\|x\|) .
$$


Observe that Assumption 8 is satisfied, e.g., if $u_{\mathscr{P}} \equiv 0$ on $T$ and $f(x, 0,0)$ is Lipschitz on $T$ with constant $L$. In this case, since $h(0,0)=f(0,0,0)=0$, we may choose $\gamma_{x}(r)=$ Lr.

Definition 9: A continuous function $V: X \rightarrow \mathbb{R}_{\geq 0}$ is called an ISpS Lyapunov function for system (2) on a sublevel set $Y=\{x \in X \mid V(x) \leq \ell\}$ for some $\ell>0$ if there exist functions $\underline{\alpha}, \bar{\alpha} \in \mathscr{K}_{\infty}, \alpha, \rho \in \mathscr{K}$, a value $\bar{w} \in \mathbb{R}_{>0}$, and a set $T \subset Y$ such that for all $x \in Y \backslash T$ the inequalities and implications

$$
\underline{\alpha}(\|x\|) \leq V(x) \leq \bar{\alpha}(\|x\|)
$$

and

$$
V(x) \geq \rho(\|w\|) \Rightarrow V(h(x, w))-V(x) \leq-\alpha(\|x\|)
$$

hold for all $w \in W$ with $\|w\| \leq \bar{w}$.

The next theorem now shows that the existence of an ISpS Lyapunov function implies ISpS and specifies the resulting gain $\gamma$ and the size $\delta$ in the practical stability definition.

Theorem 10: Consider system (2) satisfying Assumptions 7 and 8 and assume that the system admits an ISpS Lyapunov function $V$. Let $a=\sup _{x \in T}\|x\|$. Then the system is ISpS on $Y$ with

$$
\begin{aligned}
\delta & =\underline{\alpha}^{-1} \circ \bar{\alpha}\left(\max \left\{2 \gamma_{x}(a), a\right\}\right), \\
\gamma(r) & =\underline{\alpha}^{-1} \circ \bar{\alpha}\left(\max \left\{2 \gamma_{w}(r), \underline{\alpha}^{-1}(\rho(r))+\gamma_{w}(r)\right\}\right)
\end{aligned}
$$

and $\Delta_{w}=\gamma^{-1}\left(\underline{\alpha}^{-1}(\ell)\right)$ provided $\delta \leq \underline{\alpha}^{-1}(\ell)$ holds.

Proof: We fix $x_{0} \in Y, \mathbf{w} \in \mathscr{W}$ and denote the corresponding trajectory of (2) by $x_{k}$. We begin the proof by deriving estimates for $V\left(x_{k}\right)$ under different assumptions. To this end, we distinguish four different cases.

Case 1: Let $k^{\prime} \in \mathbb{N}$ be such that $V\left(x_{k}\right) \geq \rho\left(\|\mathbf{w}\|_{\infty}\right)$ and $x_{k} \in Y \backslash T$ for all $k=0, \ldots, k^{\prime}-1$. Then (17) yields

$$
\|x(k)\| \geq \bar{\alpha}^{-1}(V(x(k)))
$$

and from (18) we obtain

$$
\begin{aligned}
V\left(x_{k+1}\right)-V\left(x_{k}\right) & \stackrel{(18)}{\leq} \\
& \stackrel{(19)}{\leq}-\alpha\left(\left\|x_{k}\right\|\right) \\
& -\alpha\left(\bar{\alpha}^{-1}\left(V\left(x_{k}\right)\right)\right) .
\end{aligned}
$$

Note that $x_{0} \in Y$ and the definition of $Y$ implies $x_{k} \in Y$ for all $k=0, \ldots, k^{\prime}-1$, hence (18) may indeed be used for all these $k$. Setting $\widetilde{\alpha}:=\alpha \circ \bar{\alpha}^{-1}$, Lemma 4.3 from [18] then yields the existence of $\tilde{\beta} \in \mathscr{K} \mathscr{L}$ such that

$$
V(x(k)) \leq \tilde{\beta}\left(V\left(x_{0}\right), k\right) \text { for all } k=0, \ldots, k^{\prime} .
$$

Case 2: Let $k \in \mathbb{N}$ be such that $V\left(x_{k}\right)<\rho\left(\|\mathbf{w}\|_{\infty}\right)$ and $x_{k} \in Y \backslash T$. This implies

$$
\underline{\alpha}\left(\left\|x_{k}\right\|\right) \leq V(x(k))<\rho\left(\|\mathbf{w}\|_{\infty}\right) .
$$

and since (18) implies $V\left(h\left(x_{k}, 0\right)\right) \leq V\left(x_{k}\right)$ we get

$$
\left\|h\left(x_{k}, 0\right)\right\| \leq \underline{\alpha}^{-1}\left(\rho\left(\|\mathbf{w}\|_{\infty}\right) .\right.
$$

Then Assumption 7 implies

$$
\begin{aligned}
\left\|x_{k+1}\right\| & \leq\left\|h\left(x_{k}, 0\right)\right\|+\gamma_{w}\left(\|\mathbf{w}\|_{\infty}\right) \\
& \leq \underline{\alpha}^{-1}(\rho(\|\mathbf{w}\|))+\gamma_{w}\left(\|\mathbf{w}\|_{\infty}\right)=: \widehat{\gamma}\left(\|\mathbf{w}\|_{\infty}\right) .
\end{aligned}
$$

This yields

$$
V\left(x_{k+1}\right) \leq \bar{\alpha}\left(x_{k+1}\right) \leq \bar{\alpha}\left(\widehat{\gamma}\left(\|\mathbf{w}\|_{\infty}\right)\right)=: \widetilde{\gamma}\left(\|\mathbf{w}\|_{\infty}\right) .
$$

Case 3: Let $k \in \mathbb{N}$ be such that $V\left(x_{k}\right)<\widetilde{\gamma}\left(\|\mathbf{w}\|_{\infty}\right)$ and $x_{k} \in Y \backslash T$. Then we either have $V\left(x_{k}\right)<\rho\left(\|\mathbf{w}\|_{\infty}\right)$ and thus Case 2 implies $V\left(x_{k+1}\right) \leq \widetilde{\gamma}\left(\|\mathbf{w}\|_{\infty}\right)$.

Otherwise, we have $V\left(x_{k}\right) \geq \rho(\|\mathbf{w}\|)$ and (18) yields

$$
V\left(x_{k+1}\right) \leq V\left(x_{k}\right) \leq \widetilde{\gamma}\left(\|\mathbf{w}\|_{\infty}\right) .
$$

Thus, in either case we get $V\left(x_{k+1}\right) \leq \widetilde{\gamma}\left(\|\mathbf{w}\|_{\infty}\right)$.

Case 4: Let $k \in \mathbb{N}$ be such that $x_{k} \in T$. Then, from Assumptions 7 and 8 we get

$$
\begin{aligned}
\left\|x_{k+1}\right\| & =\left\|h\left(x_{k}, w_{k}\right)\right\| \\
& \leq\left\|h\left(x_{k}, 0\right)\right\|+\left\|h\left(x_{k}, w_{k}\right)-h\left(x_{k}, 0\right)\right\| \\
& \leq \gamma_{x}\left(\left\|x_{k}\right\|\right)+\gamma_{w}\left(\|\mathbf{w}\|_{\infty}\right) .
\end{aligned}
$$

Defining $a:=\sup _{x \in T}\|x\|$ we can continue

$$
\left\|x_{k+1}\right\| \leq \gamma_{x}(a)+\gamma_{w}\left(\|\mathbf{w}\|_{\infty}\right) \leq \max \left\{2 \gamma_{x}(a), 2 \gamma_{w}\left(\|\mathbf{w}\|_{\infty}\right)\right\}
$$

and using (17) we get

$$
\begin{aligned}
V\left(x_{k+1}\right) & \leq \bar{\alpha}\left(\max \left\{2 \gamma_{x}(a), 2 \gamma_{w}\left(\|\mathbf{w}\|_{\infty}\right)\right\}\right) \\
& =\max \left\{\bar{\alpha}\left(2 \gamma_{x}(a)\right), \bar{\alpha}\left(2 \gamma_{w}\left(\|\mathbf{w}\|_{\infty}\right)\right)\right\} .
\end{aligned}
$$

Combining these four cases we can now prove the desired inequality (3):

Let $k^{\prime} \in \mathbb{N}$ be maximal such that the condition from Case 1 is satisfied. Then, for all $k=0, \ldots, k^{\prime}$ we get

$$
\begin{aligned}
& \left\|x_{k}\right\| \stackrel{(17)}{\leq} \underline{\alpha}^{-1}\left(V\left(x_{k}\right)\right) \stackrel{(22)}{\leq} \underline{\alpha}^{-1}\left(\beta_{\widetilde{\alpha}}\left(V\left(x_{0}\right), k\right)\right) \\
& \stackrel{(17)}{\leq} \underline{\alpha}^{-1}\left(\beta_{\widetilde{\alpha}}\left(\bar{\alpha}\left(\left\|x_{0}\right\|\right), k\right)\right) \\
& =: \quad \beta\left(\left\|x_{0}\right\|, k\right)
\end{aligned}
$$

Now, for all $k \geq k^{\prime}$ by induction we show the inequality

$$
V\left(x_{k}\right) \leq \max \left\{\bar{\alpha}\left(2 \gamma_{x}(a)\right), \bar{\alpha}(a), \bar{\alpha}\left(2 \gamma_{w}\left(\|\mathbf{w}\|_{\infty}\right)\right), \widetilde{\gamma}\left(\|\mathbf{w}\|_{\infty}\right)\right\} .
$$

Note that the bounds on $\delta$ and $\Delta_{w}$ in the assertion ensure that (25) implies $V\left(x_{k}\right) \leq \ell$ and thus $x_{k} \in Y$ for all $\mathbf{w} \in \mathscr{W}$ with $\|\mathbf{w}\|_{\infty} \leq \Delta_{w}$. Hence, (25) implies that one of the Cases 1-4 must hold for $x_{k}$. Consequently, if we know that (25) holds we can use the estimates in the Cases 1-4 in order to conclude an inequality for $x_{k+1}$.

To start the induction at $k=k^{\prime}$, one checks that by definition $\widetilde{\gamma}(r) \geq \rho(r)$ holds, hence (25) follows from the condition in Case 1 and the maximality of $k^{\prime}$.

For the induction step $k \rightarrow k+1$, assume that (25) holds for $x_{k}$. Then, either Case 1 holds implying $V\left(x_{k+1}\right) \leq V\left(x_{k}\right)$ and thus (25) for $x_{k+1}$. Otherwise, one of the Cases 2-4 must hold for $x_{k}$ which also implies (25) for $x_{k+1}$.

Together, (24) and (25) show that either $\left\|x_{k}\right\| \leq \beta\left(\left\|x_{0}\right\|, k\right)$ or $\left\|x_{k}\right\| \leq \max \left\{\gamma\left(\|\mathbf{w}\|_{\infty}\right), \delta\right\}$ holds, which shows the desired ISpS inequality (3).

The following proposition shows that $V_{\mathscr{P}}$ when computed from (4), (14) is an ISpS Lyapunov function for (2).

Proposition 11: Consider the system (1), the function $V_{\mathscr{P}}$ satisfying Theorem 4 for system (4) with $\tilde{f}$ from (14) and 
the corresponding feedback $u_{\mathscr{P}}$ from (13). Then $V_{\mathscr{P}}$ is an ISpS Lyapunov function for the closed loop system (2) for any $\ell>0$.

Proof: Proof of (17): Obviously, $V_{\mathscr{P}}(x) \geq \inf _{u \in U} g(x, u)$ if $x \notin T$. Hence the lower bound in (17) follows directly from Assumption 3. The existence of an upper bound follows since $V_{\mathscr{P}} \equiv 0$ holds on $T$ and $V_{\mathscr{P}}$ is piecewise constant and bounded by $\ell$ on $Y$. Hence, $\sup _{x \in Y,\|x\| \leq r} V_{\mathscr{P}}(x)$ is piecewise constant, finite for each $r>0$ and equal to 0 for all sufficently small $r>0$. Thus, it can be overbounded by a function $\bar{\alpha} \in$ $\mathscr{K}_{\infty}$ (which could, e.g., be constructed by piecewise linear interpolation).

Proof of (18): From (12) and the definition of $u_{\mathscr{P}}$ via (13) it follows that $V_{\mathscr{P}}(x) \geq g\left(x, u_{\mathscr{P}}(x)\right)+V_{\mathscr{P}}(\tilde{h}(x, d))$ holds for all $x \in Y \backslash T$ and all $d \in D$. Since by assumption on $e$ in (14) for all $w \in W$ with $\|w\| \leq \eta(\|x\|)$ we find $d \in D$ with $w=e(x, d)$, this implies that for all such $w$ we obtain the right inequality in (18). This shows that (18) holds with $\rho=\eta$.

Note that since $V_{\mathscr{P}}$ assumes only finitely many different values and is finite on $S_{\mathscr{P}}$, choosing $\ell:=\max _{x \in S_{\mathscr{P}}} V_{\mathscr{P}}(x)$ yields the maximal possible domain $Y=S_{\mathscr{P}}$ on which $V_{\mathscr{P}}$ is an ISpS Lyapunov function.

Our final and main result now summarizes the conditions under which the feedback $u_{\mathscr{P}}$ indeed renders System (1) ISpS.

Theorem 12: Consider a system (1) satisfying Assumptions 7 and 8, the function $V_{\mathscr{P}}$ satisfying Theorem 4 for system (4) with $\tilde{f}$ from (14) and the corresponding feedback $u_{\mathscr{P}}$ from (13). Let $\ell \leq \max _{x \in S_{\mathscr{P}}} V_{\mathscr{P}}(x)$ and let $\underline{\alpha}, \bar{\alpha} \in \mathscr{K}_{\infty}$ denote functions such that (17) holds ${ }^{2}$ on $Y=\left\{x \in X \mid V_{\mathscr{P}}(x) \leq \ell\right\}$.

(i) If the value $\ell>0$ is such that the inequality

$$
\ell \geq \bar{\alpha}\left(\max \left\{2 \gamma_{x}(a), a\right\}\right)
$$

for $a=\sup _{x \in T}\|x\|$ holds, then the system is ISpS on $Y$ w.r.t $\delta=\underline{\alpha}^{-1} \circ \bar{\alpha}\left(\max \left\{2 \gamma_{x}(a), a\right\}\right)$ and $\Delta_{w}$ as specified in Theorem 10 .

(ii) If for each target $T$ which forms a neighborhood of 0 there exists a partition $\mathscr{P}$ such that $\bar{\alpha}$ in (17) can be chosen independently of $T$, then for each $\delta>0$ there exists $T$ and $\mathscr{P}$ such that $u_{\mathscr{P}}$ is ISpS on $Y$ w.r.t. this $\delta$ and $\Delta_{w}$ as specified in Theorem 10.

Proof: (i) By Proposition 11 the function $V_{\mathscr{P}}$ is an ISpS Lyapunov function. Since (26) ensures that Theorem 10 is applicable, this yields the ISpS property.

(ii) By choosing $T$ to be a sufficiently small neighborhood around 0 we can choose $a=\sup _{x \in T}\|x\|$ arbitrarily close to 0 . Since, moreover, $\bar{\alpha}$ is independent of the choice of $T$ (and by the first part of the proof of Proposition $11 \underline{\alpha}$ is independent of $T$, too) we can always ensure that (26) holds and that $\delta$ as specified in (i) can be chosen arbitrarily small. This shows the assertion.

Remark 13: Under suitable conditions it is reasonable to expect that the condition on $\bar{\alpha}$ from Theorem 12(ii) holds

\footnotetext{
${ }^{2}$ These functions exist according to the first part of the proof of Proposition 11.
}

provided the partition $\mathscr{P}$ is - at least near the target $T$ sufficiently fine. This is due to the fact that with similar arguments as in the proofs of [13, Theorem 4.3] or [12, Theorem 5.4] we can first ensure that such a bound holds for $V$. In the next step, using similar arguments as in [9], by choosing the partition $\mathscr{P}$ sufficiently fine one can ensure that $V_{\mathscr{P}}$ is close to $V$ from which the assertion follows. Note that the smaller the target $T$ is the smaller the error and thus the finer the partition $\mathscr{P}$ must be in order to make this argument work. Details about this construction will be investigated in future research.

Remark 14: Since the computational part of our approach entirely relies on computing a uniformly practically asymptotically stabilizing feedback law for the scaled system (4) by means of (a slight generalization of) the approach from [8], all the extensions developed for this algorithm can be readily applied to our proposed approach. Particularly, this concerns the approach to implement the computation of feedback laws depending not only on the current but also on past values of the state [10] and an event based computation of $u_{\mathscr{P}}$ [11]. Both extensions together allow for a significant reduction of the number of partition elements representing $u_{\mathscr{P}}$. They have thus been used in the computations of our numerical example in the next section.

\section{NUMERICAL EXAMPLE}

In order to illustrate our approach we show numerical results for a thermofluid process. The process consists of the fill level $x_{1}$ and the temperature $x_{2}$ of a liquid in a tank. The inflow of liquid can be controlled by $u_{1}$ and the liquid in the tank can be cooled using $u_{2}$. The perturbations of the water level $w_{1}$ and the temperature $w_{2}$ model the unknown inflow of liquid from a second tank. For a description of the full model see [21, Appendix]. After some simplifications of the equations, the behavior of the tank system is described by the state-space model

$$
\begin{aligned}
\dot{x}_{1}(t)=\frac{1}{0.065}\left(161 \cdot 10^{-6} u_{1}(t)+129 \cdot 10^{-6} \sqrt{w_{1}(t)+0.34}\right. \\
\left.-270 \cdot 10^{-6} \sqrt{x_{1}(t)}\right) \\
\dot{x}_{2}(t)=\frac{1}{0.065 x_{1}(t)}\left(129 \cdot 10^{-6} \sqrt{w_{1}(t)+0.34}\right. \\
\times\left(w_{2}(t)+300-x_{2}(t)\right) \\
\left.+97 \cdot 10^{-6} u_{2}(t)\left(287-x_{2}(t)\right)\right)
\end{aligned}
$$

with $X=[0.25,0.4] \times[290,320], w_{1} \in[-0.09,0.09], w_{2} \in$ $[-20,20]$ and $u_{i} \in[0,1], i=1,2$. For $u^{\star}=$ $(0.481465,0.48466)^{T}$, the equation exhibits the equilibrium $x^{\star}=(0.32,295)^{T}$. Note that $x^{\star}$ is asymptotically stable, hence the goal of our ISpS controller is not to stabilize the system at $x^{\star}$ but to increase the robustness of the stability against perturbations.

As the system has a cascaded (or triangular) structure i.e., the first equation does not depend on $x_{2}-$ it has turned out beneficial to choose $e$ in (14) to reflect this structure, i.e., to have the first component independent of $x_{2}$. Note that this 
way the assumption needed in the second part of the proof of Proposition 11 is not satisfied; however, the proof can be adapted to the cascaded situation. Hence we chose $e$ in (14) as

$$
e(x, d)=\left(\begin{array}{c}
\sqrt{1.25297\left(x_{1}-x_{1}^{\star}\right)^{2}} d_{1} \\
\sqrt{618.75\left(x_{1}-x_{1}^{\star}\right)^{2}+0.6273\left(x_{2}-x_{2}^{\star}\right)^{2}} d_{2}
\end{array}\right)
$$

We computed the controller using the stage cost $g(x, u)=$ $4 \cdot 10^{4}\left(x_{1}-x_{1}^{\star}\right)^{2}+\left(x_{2}-x_{2}^{\star}\right)^{2}$ on a partition $\mathscr{P}$ consisting of $32 \times 32$ equally sized elements where the target $T$ was chosen as the partition element containing $x^{\star}$. The sampling time was chosen as $2 s$, and the control and perturbation value sets $U=[0,1]^{2}$ and $D=[-1,1]^{2}$ were discretized with grids of $9 \times 5$ and $3 \times 3$ equidistant nodes, respectively.

For the trajectory simulations a randomly generated sequence $\mathbf{w}$ of perturbations was used, using uniformly distributed random numbers in $[0,0.09]$ and $[0,20]$, respectively, for the components of each vector $w_{k} \in \mathbb{R}^{2}$. The resulting trajectories with and without control (both for the same sequence w) are shown in Figure 1. One clearly sees that the controller is able to bring the system considerably closer to the desired equilibrium. However, the practical nature of the controller is also clearly visible by the zig-zagging effect of the $x_{1}$-component. This effect could probably be reduced by using a local robust controller near $x^{\star}$ instead of the constant equilibrium control value $u^{\star}$ we have employed in our simulation.
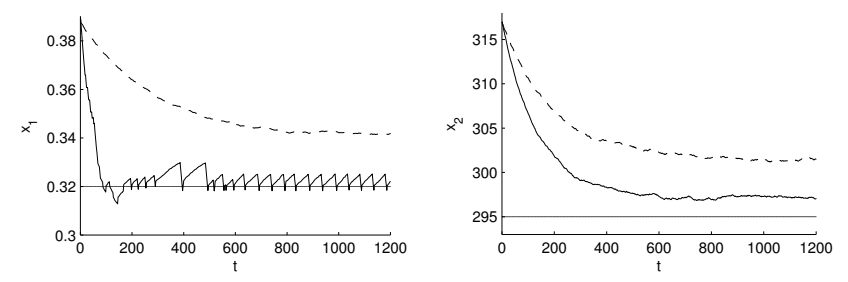

Fig. 1. Trajectories with control (solid) and without control (dashed), $x_{1}$ component (left) and $x_{2}$-component (right)

\section{CONCLUSIONS AND OUTLOOK}

We have shown that the equivalence between ISS and robust stability proved in [17] for discrete time systems can be used as the basis for a numerical approach for computing ISS feedback laws. As the underlying algorithm for calculating uniformly stabilizing feedback laws from [8] yields only practically stabilizing controllers, the resulting feedback law will be input-to-state practically stabilizing w.r.t. some $\delta>0$. In order to obtain a meaningful stability property, a careful analysis of the size $\delta$ of the practical stability region is provided in this paper. As Theorem 12 reveals, the existence of an upper bound $\bar{\alpha}$ on $V_{\mathscr{P}}$ which is independent of the target set $T$ is a crucial property for bounding $\delta$.

The rigorous derivation of such upper bounds will be investigated in future research, cf. Remark 13. Moreover, we intend to address the extension of our analysis to event based ISpS feedback laws and the proposed computational approach for ISpS controllers will be used as a building block for a distributed feedback design for large networks of systems based on the small gain arguments from [2], [3].

\section{REFERENCES}

[1] D. P. Bertsekas, Dynamic Programming and Optimal Control. Vol. 1 and 2., Athena Scientific, Belmont, MA, 1995.

[2] S. N. DAShKovSKIY, B. S. RÜFFER, AND F. R. Wirth, An ISS small gain theorem for general networks., Math. Control Signals Syst., 19 (2007), pp. 93-122.

[3] S. N. DAShKovskiY, B. S. RÜFFER, AND F. R. Wirth, Small gain theorems for large scale systems and construction of ISS Lyapunov functions, SIAM J. Control Optim., 48 (2010), pp. 4089-4118.

[4] P. GIESL, Construction of global Lyapunov functions using radial basis functions, vol. 1904 of Lecture Notes in Mathematics, Springer, Berlin, 2007.

[5] P. GIESL AND S. HAFSTEIN, Existence of piecewise linear Lyapunov functions in arbitrary dimensions, Discrete Contin. Dyn. Syst., 32 (2012), pp. 3539-3565.

[6] L. Grüne, S. Jerg, O. Junge, D. Lehmann, J. Lunze, F. MÜLlER, AND M. Post, Two complementary approaches to event-based control, at-Automatisierungstechnik (Special Issue on Networked Control Systems), 58 (2010), pp. 173-182.

[7] L. GRÜNE AND O. JUNGE, Optimal stabilization of hybrid systems using a set oriented approach, in Proceedings of the 17th International Symposium on Mathematical Theory of Networks and Systems MTNS2006, Kyoto, Japan, 2006, pp. 2089-2093.

[8] L. GRÜNE AND O. JUNGE, Approximately optimal nonlinear stabilization with preservation of the Lyapunov function property, in Proceedings of the 46th IEEE Conference on Decision and Control, New Orleans, Louisiana, 2007, pp. 702-707.

[9] L. GRÜNE AND O. JUNGE, Global optimal control of perturbed systems, J. Optim. Theory Appl., 236 (2008), pp. 411-429.

[10] L. GRÜNE AND F. MÜLlER, Set oriented optimal control using past information, in Proceedings of the 18th International Symposium on Mathematical Theory of Networks and Systems (MTNS2008), Blacksburg, Virginia, 2008. CD-ROM, Paper 125.pdf, 6 pages.

[11] L. GRÜNE AND F. MÜLLER:, An algorithm for event-based optimal feedback control, in Proceedings of the 48th IEEE Conference on Decision and Control, Shanghai, China, 2009, pp. 5311-5316.

[12] L. GRÜNE AND D. NešIĆ, Optimization based stabilization of sampled-data nonlinear systems via their approximate discrete-time models, SIAM J. Control Optim., 42 (2003), pp. 98-122.

[13] L. Grüne And J. Pannek, Nonlinear Model Predictive Control. Theory and Algorithms, Springer-Verlag, London, 2011.

[14] S. F. HAFSTEIN, An algorithm for constructing Lyapunov functions, vol. 8 of Electronic Journal of Differential Equations. Monograph, Texas State University-San Marcos, Department of Mathematics, San Marcos, TX, 2007. Available electronically at http://ejde.math.txstate.edu/.

[15] S. HuAng, M. R. JAmes, D. NešIĆ, AND P. M. Dower, A unified approach to controller design for achieving ISS and related properties, IEEE Trans. Automat. Control, 50 (2005), pp. 1681-1697.

[16] Z. P. Jiang, A. R. TEel, AND L. Praly, Small-gain theorem for ISS systems and applications, Math. Control Signals Syst., 7 (1994), pp. 95-120.

[17] Z.-P. JIANG AND Y. WANG, Input-to-state stability for discrete-time nonlinear systems., Automatica, 37 (2001), pp. 857-869.

[18] Z.-P. JIANG AND Y. WANG, A converse Lyapunov theorem for discrete-time systems with disturbances, Systems Control Lett., 45 (2002), pp. 49-58.

[19] O. JUNGE AND H. M. OsINGA, A set oriented approach to global optimal control, ESAIM Control Optim. Calc. Var., 10 (2004), pp. 259270.

[20] D. Liberzon, E. D. SonTAG, AND Y. WANG, Universal construction of feedback laws achieving ISS and integral-ISS disturbance attenuation, Systems Control Lett., 46 (2002), pp. 111-127.

[21] J. LunZE, ed., Control Theory of Digitally Networked Systems, Springer, 2013. To appear.

[22] E. D. Sontag, Smooth stabilization implies coprime factorization, IEEE Trans. Autom. Control, 34 (1989), pp. 435-443.

[23] E. D. Sontag AND Y. WANG, New characterizations of input-tostate stability, IEEE Trans. Autom. Control, 41 (1996), pp. 1283-1294. 